\title{
Evaluación de métricas del comportamiento del tráfico de video en una red experimental multidifusión
}

\section{(Evaluation of video traffic behavior metrics in an experimental multicast network)}

\author{
Higinio Alberto Facchini, ${ }^{1}$ Santiago Cristóbal Pérez, ${ }^{2}$ Alejandro Dantiacq, ${ }^{3}$ Fabián Hidalgo ${ }^{4}$
}

\begin{abstract}
Resumen
En la actualidad, se observa un cambio importante en el tráfico de datos en las redes, tanto locales como de Internet, en todo el espectro de clientes posibles, sean estos fijos o móviles. El consumo de tráfico de video se incrementa día a día en todos sus formatos. A los proveedores de servicios se les exige mayor ancho de banda, calidad y rendimiento, para los todos los productos, pero fundamentalmente a los basados en video. En este artículo se presenta una versión mejorada de nuestros ensayos de laboratorio, para redes genéricas, con cuatro sub-escenarios, que permiten inyectar tráfico de video de tipo multidifusión, representado con tres diferentes codecs. La topología de red utilizada incluye un equipo servidor de streaming, y hasta 20 computadoras de escritorio como clientes. Se definieron las métricas más importantes a analizar, basadas en el desempeño y la calidad de servicio (QoS), bajo condiciones de ancho de banda limitado. Los resultados numéricos experimentales pueden ser de utilidad para los administradores, analistas de simulación, diseñadores, y planificadores de redes de datos. Pero, además, este documento pretende servir como guía para la gestión de redes similares, y para utilizar eficientemente los recursos disponibles, sin comprometer el desempeño y QoS.
\end{abstract}

\section{Palabras clave}

Laboratorio experimental WAN; tráfico video; codec de video; QoS; tráfico multidifusión.

\begin{abstract}
Currently, there is a significant change in data traffic on networks, both local and Internet, across the spectrum of possible customers, whether fixed or mobile. The consumption of video traffic increases day by day in all its formats. Service providers are required more bandwidth, quality and performance, for all products, but mainly those based on video. This article presents an improved version of our laboratory tests for generic networks with four sub-scenarios, which allow injecting multicast video traffic, represented in different codecs. The topology of network used includes a streaming server and up to 20 desktop computers as clients. The most important metrics to be analyzed have been defined, based on performance and quality of service (QoS) measurements under limited bandwidth conditions. Experimental numerical results can be useful for administrators, simulation analysts, designers, and data network planners. But, also, this document aims to serve as a guide for the management of similar networks and the efficiently use available resources, without compromising performance and QoS.
\end{abstract}

\section{Keywords}

WAN test bed; video traffic; video codec; QoS; multicast traffic.

\section{Introducción}

Anualmente, la empresa Cisco elabora su reporte Cisco Visual Networking Index (2019), en el que se discuten los efectos del tráfico interredes global, también se presentan algunas de las conclusiones principales del tráfico IP global proyectado, además se exploran las implicaciones del crecimiento del tráfico IP para proveedores de servicios. Las principales conclusiones observadas, incluyen: 
- El tráfico IP global anual llegará a 3,3 ZB por año hasta el año 2021 (en 2016, la tasa anual para el tráfico IP global fue de 1.2 ZB por año).

- El tráfico IP mensual alcanzará los 35 GB per cápita en 2021, frente a los 13 GB per cápita en 2016.

- El tráfico de datos de dispositivos móviles (smartphones) superará el tráfico correspondiente a puestos de trabajo cableados (PCs de escritorio).

- El número de dispositivos conectados a redes IP será más de tres veces la población mundial para el 2021.

- Las velocidades de datos, en banda ancha, llegará prácticamente a duplicarse en 2021.

Al hablar específicamente sobre el tráfico de video IP global, se proyecta que constituirá el 82 \% de todo el tráfico IP en 2021 y crecerá el triple, de 2016 a 2021. El video por Internet continuará creciendo a un ritmo acelerado, aumentando 3.6 veces durante el mismo período. Este reporte de Cisco (2019) plantea que la suma de todas las formas de video IP, incluyendo video por Internet, Video bajo demanda (VoD), archivos de video IP intercambiados a través de métodos de movimientos de archivos, streaming de video, juegos y videoconferencias, permanecerá en el rango de $80 \%$ a $90 \%$ del tráfico total IP.

Por lo tanto, entendemos que un buen conocimiento sobre el tráfico de video, de redes multimedia en multidifusión, sobre la codificación o compresión de video, y los requisitos de la red, resultará de mucha utilidad para los administradores, analistas por simulación, diseñadores y planificadores de redes de datos.

Cuando se trata de tráfico multimedia, la multidifusión y la compresión son dos herramientas importantes para mitigar el impacto de la creciente carga de tráfico de video en una red.

La multidifusión IP es una técnica encaminada a conservar el ancho de banda, específicamente diseñada para reemplazar varios flujos de datos enviados (uno para cada receptor) con la entrega de un único flujo de información. Por lo tanto, una dirección IP multidifusión contribuye a minimizar el volumen de carga, tanto en los hosts de origen y de destino y, al mismo tiempo, reducir el tráfico global de la red.

La compresión de vídeo reduce la cantidad de datos necesarios para transmitir o almacenar señales de video digital. Una serie de estándares han sido desarrollados para la codificación de video, considerando distintas técnicas de compresión, y logrando que algunos codecs realicen esta función de manera más eficiente que otros, puesto que la compresión de vídeo puede realizarse utilizando distintas técnicas:

- compresión intratrama: analiza de forma independiente cada una de las tramas de vídeo y realiza un proceso de compresión de cada una de ellas sin tener en cuenta similitudes o parecidos con las tramas próximas.

- compresión intertrama: analiza el parecido entre las diferentes tramas contiguas y realiza un proceso de compresión de cada una de ellas, teniendo en cuenta similitudes o parecidos con dichas tramas próximas.

Una variedad de estudios pone a consideración diversas conclusiones sobre estos temas, entre ellos; el análisis del tráfico de multidifusión y los protocolos de enrutamiento de multidifusión 
(Ueno, Kato y Suzuki, 2000; Hosseini, Tanvir, Shirmohammadi y Georganas, 2007; Kenji, Masataka y Katsuo, 2000; Brown, Crowcroft, Handley y Cain, 2002), que sirve para comprender las características de tráfico de multidifusión y su comportamiento en la red. Otros trabajos hacen que los codecs de vídeo, más allá de las normas conocidas, permitan comprender su comportamiento (Divxland 2012; Abdalla, Mazhar, Salah y Khalaf, 2014); y a los artículos referidos sobre el tráfico de vídeo en diferentes contextos (Grois, Marpe, Nguyen y Hadarb, 2014; Klare y Burge, 2010; Sun, Sheriff, Belding-Royer y Almeroth, (2005); Addu y Potuvardanam, 2014; Lazaris y Koutsakis, 2010; Tanwir y Perros, 2013; Dai, Zhang y Loguinov, 2009; Auwera, David y Reisslein, 2008; Seeling, Reisslein y Kulapala, 2004; Shin et al. 2011; Egger y Braun, 2003; Chandrasekar y Baskaran, 2012; Cachinero, 2009), que permiten evaluar el amplio espectro de estudios existentes sobre tráfico de vídeo.

Para complementar las referencias, se mencionan artículos relacionados al análisis de QoS para el tráfico de video (Tanwir, Perros y Anjum, 2014; Perez et al. 2015) y estudios sobre IPTV (Nakayama, Watanabe y Higashi, 2008; Torres, Ramírez y López, 2011) que plantean un caso particular de tráfico de video.

Al considerar parámetros de medición para conocer y comprender el comportamiento de las redes, es importante ver otros artículos no relacionados directamente con el tráfico de vídeo. Se pueden mencionar el análisis de una red inalámbrica para VoIP (Villarreal, Mariño, Márquez y Núñez, 2019) y el estudio de la sobrecarga de una red en redes móviles (Ludeña, Torres, Quiñonez y Cueva, 2017).

Los trabajos de investigación relacionados con la materia muestran, en general, la falta de uniformidad de las situaciones experimentales, en el número y tipos de codecs, en los videos utilizados, etc. Estos y otros aspectos pueden complicar, como un todo, los contrastes entre las obras contemporáneas entre sí, y las realizadas anteriormente.

Con este documento se pretende llegar a nuevas conclusiones cuantitativas en los estudios experimentales sobre las mejores opciones, para el tráfico de video en redes de laboratorios de ambientes controlados, con equipamiento real. La experiencia corresponde a un caso específico de un vídeo bajo demanda de baja resolución, pregrabado, de 29 segundos de duración y con bajos requisitos de carga, que se inicia en los momentos especificados en una red WAN con hasta 20 usuarios, como clientes simultáneos.

Las principales contribuciones de este trabajo son: (i) demostrar, basándose en nuevos resultados cuantitativos detallados, que todas las métricas de rendimiento y calidad de servicio (QoS) obtenidos y analizados son los esperados para el tráfico de video de multidifusión, y que dependen exclusivamente del codec de video utilizado, y no sobre la topología de la red o el número de clientes que reciben video streaming; (ii) la especificación de un nuevo ensayo experimental compuesto de cuatro escenarios, y de una nueva metodología con mecanismos comparativos, a fin de determinar las diferencias entre los escenarios; y (iii) la determinación experimental del comportamiento de codec.

El resto de este documento está estructurado de la siguiente manera: en la sección 2 se presenta la situación y los recursos de la experimentación; la sección 3 presenta sus resultados; y, finalmente, en la sección 4 se presentan las conclusiones más importantes.

\section{Metodología}

Este estudio pretende evaluar experimentalmente el rendimiento de un flujo de un vídeo pregrabado bajo demanda, en un laboratorio experimental de ambiente controlado, que permite 
simular una red WAN, y que utiliza equipos reales de red, como switchs y routers, servidores de streaming y clientes receptores.

La topología genérica que se ha utilizado incluye una computadora, que funciona como un servidor de streaming, y 20 computadoras de escritorio (PCs), trabajando como clientes, conectados en los extremos de la topología, como si se tratara de una red real del tipo WAN. Esta red está formada por los dispositivos mencionados, con diferentes tipos de vínculos, teniendo una topología redundante en cuanto a enlaces, para su semejanza con redes reales de cierta envergadura. La figura 1 muestra la topología general.

En esta topología, los vínculos representados por líneas sólidas pertenecen al tipo FastEthernet, con una velocidad de transmisión de 100 Mbps, y los vínculos representados por líneas discontinuas están asociados a las interfaces seriales, para conexiones punto a punto, con velocidades de transmisión de 4 Mbps.

El núcleo de la red tuvo como funcionalidad principal el ruteo de todos los tráficos, sobre el que se realizaron las configuraciones de los distintos caminos del tráfico. Para dicha funcionalidad, entre los routers R1 a R6 se estableció el protocolo de enrutamiento de tipo unidifusión OSPF v2. Para el caso de multidifusión, se estableció el protocolo de enrutamiento multidifusión PIM de modo denso. Los enlaces redundantes existentes no solo se presentan como una opción para simular una WAN, sino también como un medio para configurar el protocolo de enrutamiento, para el tráfico entre el servidor y cada cliente, siguiendo una ruta única en todo momento.

Figura 1. Topología utilizada

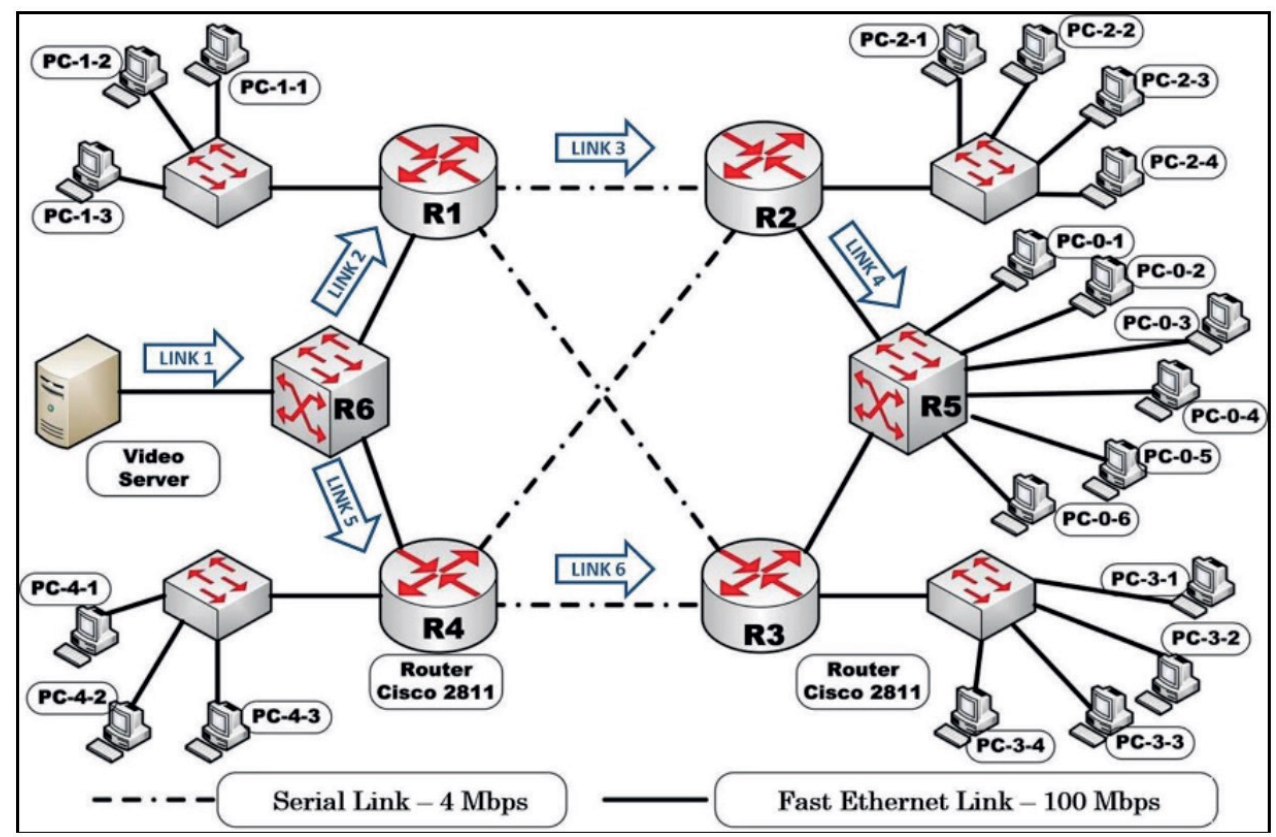

La topología presentada se desarrolló utilizando el siguiente hardware:

- Servidor: un computador de escritorio con el procesador Intel Core i5 CPU, 8 GB de RAM y Windows 7 Professional de 64 bits.

- Clientes: computadoras de escritorio con procesador AMD Athlon(tm) IIX2 250 a 3GHz, 4 GB de RAM y Windows 7 Professional de 64 bits del sistema operativo. 
- Los routers R1, R2, R3 y R4 fueron implementadas con equipos de la marca Cisco modelo 2811 y los routers R5 y R6 se implementaron con equipos de la marca Cisco modelo WS-CS3750.

- Por último, para la conexión entre los routers y las PCs, se utilizaron switchs de la marca Cisco modelo Catalyst WS-2950-24.

Los softwares de aplicación utilizados son:

- Servidor de Streaming: Unreal Media Server (Unreal), software de streaming de licencia libre (con ciertas restricciones, pero apto para el presente estudio)

- Los clientes receptores del streaming: Unreal Media Player instalado en todos los equipos cliente,

- Analizador de Tráfico: Wireshark (Wireshark), un software gratuito y de código abierto, que funciona como analizador de paquetes de red utilizados en la solución de problemas, el análisis, el desarrollo de software y protocolos de comunicación y educación.

Para el desarrollo de la experimentación, se utilizó un video comercial de 29 segundos (video base) codificado originalmente en H.264. El video se recodificó usando diferentes codecs de video:

- H.264 / MPEG-4 AVC.

- H.263 / MPEG-4 Part 2.

- MPEG-2.

Se determinó la utilización de los codecs anteriores, debido a que los mismos son típicos y muy utilizados en la codificación de video, y para diferenciar distintas tecnologías. Las configuraciones básicas de resolución, tamaño y codificación de los codecs se muestran en la tabla 1. Los cuadros presentan los parámetros característicos de los codecs y contenedores del video utilizado.

En la tabla planteada, las líneas de "Características generales" indican las propiedades del archivo contenedor, y las líneas de "Características de Vídeo" presentan las propiedades propias del vídeo. Como referencia, es válido indicar que se utilizó una resolución de vídeo relativamente baja, en la línea de formato QCIF (aproximadamente 176*144), para evaluar el comportamiento sobre los enlaces seriales típicos WAN, en la medida que crece el número de usuarios finales y se compromete su ancho de banda.

En las misma tabla, se puede observar, a priori, que existen parámetros diferentes entre los distintos codecs, con base en las características propias de cada codec en particular, y al proceso de recodificación 
Tabla 1. Parámetros del video

\begin{tabular}{|c|c|c|c|}
\hline \multicolumn{4}{|c|}{ Características generales archivo (contenedor) } \\
\hline Parámetros & H.264/MPEG-4 AVC & H.263/MPEG-4 Visual & MPEG-2 \\
\hline Duración del video & 29 seg & 29 seg & $29 \mathrm{seg}$ \\
\hline Formato Codec & MPEG-4 & MPEG-4 & MPEG-TS \\
\hline ID del codec & isom & $3 g p 6$ & TS \\
\hline Tamaño archivo & $616 \mathrm{~Kb}$ & $292 \mathrm{~Kb}$ & $724 \mathrm{~Kb}$ \\
\hline Velocidad & $169 \mathrm{Kbps}$ & 79.4 Kbps variable & 202 Kbps \\
\hline \multicolumn{4}{|c|}{ Características video } \\
\hline ID del codec & AVC 1 & MPEG-4 Visual & MPEG \\
\hline Descriptor del codec & Advanced Video Coding & H.263 & MPEG-2 \\
\hline Formato & HighaL1.1 & Simple@LO & \\
\hline Velocidad & $166 \mathrm{Kbps}$ & 53.7 Kbps variable & 192 Kbps \\
\hline Resolución & $176 \times 164$ pixeles & 176x144 pixeles & $176 \times 160$ pixeles \\
\hline Relación de aspecto & 1.734 & 1.222 & 1.1 \\
\hline Velocidad de trama & $25 \mathrm{fps}$ & $8.333 \mathrm{fps}$ & $60 \mathrm{fps}$ \\
\hline Coloración & YUV & YUV & YUV \\
\hline Profundidad de bits & 8 bits & 8 bits & 8 bits \\
\hline Tipo de scan & Progresivo & Progresivo & Progresivo \\
\hline Bits (pixel x trama) & 0.231 & 0.254 & 0.113 \\
\hline Tamaño de flujo & 607 Kb (99 \%) & 198 Kb (68 \%) & 676 Kb (93 \%) \\
\hline
\end{tabular}

En el proceso de experimentación, se aplicó un procedimiento acordado con una serie de consideraciones y secuencia de pasos comunes:

- Se utilizó el archivo de vídeo descripto codificado en 3 formatos distintos (MPEG-4/ AVC, MPEG-4 Visual o MPEG-2),

- Antes de comenzar las mediciones se sincronizaron en tiempo todos los equipos involucrados en la topología, usando un servidor NTP local.

- En primera instancia, desde el servidor se envió el archivo de video propuesto, en un codec en particular, a la red en formato multidifusión (multicast).

- Posteriormente, se cambió a los otros dos codec de video, repitiendo el paso anterior.

- Durante cada ensayo, las mediciones se realizaron capturando tráfico en el servidor y en cada una de las PCs clientes, usando el software Wireshark. Con este software se generó un archivo con la captura de todas las tramas, para cada ensayo, como se muestra en la figura 2. Con este archivo se dispone de toda la información relevante de cada una de las tramas capturadas durante el ensayo, como el día y hora exacta de la captura de cada trama, las direcciones MAC e IP origen y destino, el protocolo de capa de transporte y/o de aplicación, el tamaño de trama total y disgregado por cada campo, etc. 
- La experimentación se realizó en un ambiente controlado, con solamente tráfico de video en la red, para los efectos de obtener su real comportamiento, sin el impacto o influencia de otros tráficos de usuario. Por supuesto, que en la captura también apareció el tráfico de información de los protocolos de ruteo y otros tráficos de control de los dispositivos activos. La cantidad de paquetes de estos tráficos fue despreciable respecto a la cantidad de paquetes del tráfico de video y, por lo tanto, fueron descartados del estudio, sin que esto significara alterar los resultados numéricos de las métricas ni las conclusiones de este estudio.

Figura 2. Captura Wireshark

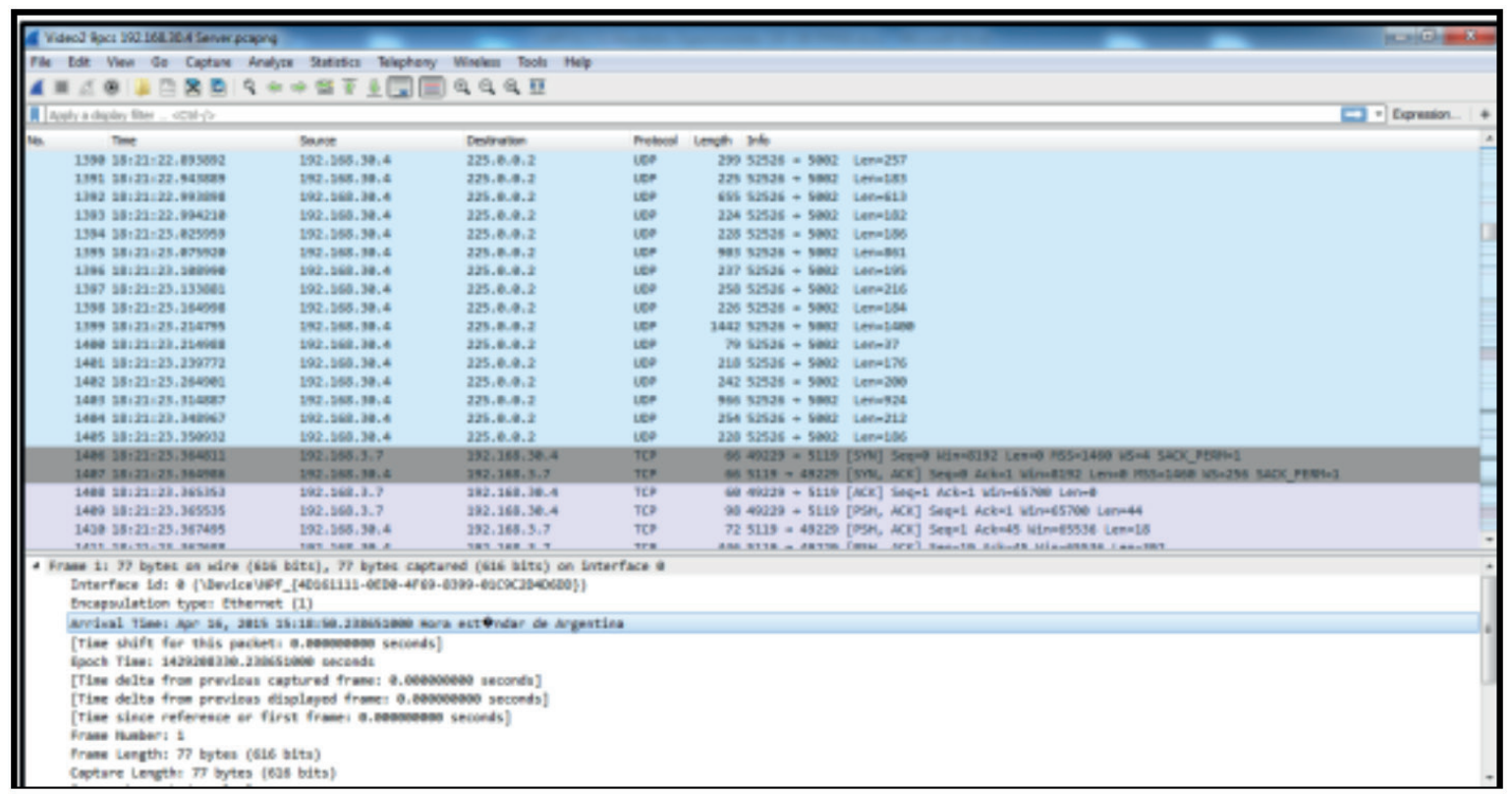

Para realizar los análisis correspondientes, se consideraron distintas métricas a evaluar:

- Tiempo total del video [Tt]: definido como el tiempo transcurrido entre la transmisión del primer y el último paquete más uno.

- Número total de paquetes (o tramas) [NP]: definido como la cantidad total de paquetes perteneciente al tráfico de video durante el tiempo Tt.

- Número total de bytes [NB]: definido como la cantidad total de bytes perteneciente al tráfico de video durante el tiempo Tt.

- Número de errores obtenidos [Eo]: definido como la cantidad de tramas recibidas con error y/o cantidad de tramas perdidas.

- Tamaño promedio de los paquetes [Tp]: definido como la suma total de bytes de todos los paquetes de video dividido por NP.

- Espacio intertrama o Tiempo entre tramas de paquetes [EI]: definido como el tiempo transcurrido entre el inicio de un paquete y el inicio del paquete siguiente. 
- Tasa de transferencia efectiva [BR]: definida como NB dividido por Tt.

- Retardos de tiempo involucrados en la transmisión [Rt]: definido como el tiempo transcurrido desde que salió el primer bit del origen hasta que ese bit llegó al destino.

- Diferencia de retardo (o jitter) [DR]: definido para 2 paquetes dados como la diferencia entre los Rt de cada uno.

- Distribuciones estadísticas de tamaño de paquete.

- Distribuciones estadísticas de tiempo entre tramas.

- Distribuciones estadísticas de segundo orden y análisis de autosimilitud.

En función de lo expuesto, se procesó cada archivo de captura para obtener cada una de las métricas definidas. Teniendo en cuenta los detalles especificados anteriormente, se adoptaron 4 escenarios de experimentación:

- Escenario 1: en la que se emite desde el servidor y se recibe solamente en tres clientes (PC2-1, PC0-1 y PC3-1).

- Escenario 2: en la que se emite desde el servidor y se recibe solamente en seis clientes (PC1-1, PC2-1, PC0-1, PC0-2, PC3-1 y PC4-1).

- Escenario 3: en la que se emite desde el servidor y se recibe en solamente en nueve clientes (PC1-1, PC2-1, PC2-2, PC0-1, PC0-2, PC0-3, PC3-1, PC3-2 y PC4-1).

- Escenario 4: en la que se emite desde el servidor y se recibe en todos los clientes.

Se aclara que, para el desarrollo de este artículo, y por cuestiones de espacio, se presentan algunas de las métricas descritas directas y promedio.

\section{Resultados y discusión}

En los ensayos globales realizados, los valores de las métricas obtenidas en forma directa, como las distribuciones estadísticas calculadas, muestran valores muy similares, independientemente de la ubicación de la PC, o si el tráfico es de unidifusión o multidifusión (para el conjunto de configuración en el laboratorio). El tiempo y sincronización de video muestra diferencias mínimas, cuando se reproducen en PCs diferentes, dependiendo del codec utilizado.

En las tablas 2, 3, 4 y 5 se resumen las mediciones obtenidas para cada escenario de las métricas discutidas en este artículo. Se muestra solamente el promedio global de cada métrica (obtenido de las mediciones realizadas en el servidor y en los clientes), ya que, como se planteó, las diferencias entre las métricas individuales son despreciables.

En las tablas se muestra una línea para cada codec, tanto para el promedio global como el para el porcentaje promedio. El porcentaje se calcula directamente del valor individual de cada codec, dividido la suma total de todos los codecs, para visualizar el impacto de cada codec en particular.

Se puede observar que los valores no varían sustancialmente, comparando cualquier escenario. Esto comprueba la hipótesis de que el tráfico multidifusión es el mismo independientemente de la cantidad de clientes conectados a un único flujo de tráfico. 
Tabla 2. Métricas escenario 1

\begin{tabular}{|l|l|l|l|l|l|l|}
\hline & Codec & $\begin{array}{c}\text { Cantidad de } \\
\text { paquetes }\end{array}$ & $\begin{array}{c}\text { Cantidad } \\
\text { de bytes } \\
\text { [Mbytes] }\end{array}$ & $\begin{array}{c}\text { Tamaño } \\
\text { promedio } \\
\text { paquetes } \\
\text { [bytes] }\end{array}$ & $\begin{array}{c}\text { Tiempo } \\
\text { promedio } \\
\text { intertrama } \\
\text { [s] }\end{array}$ & $\begin{array}{c}\text { Velocidad } \\
\text { promedio } \\
\text { [Mbps] }\end{array}$ \\
\hline \multirow{4}{*}{ Promedio general } & MPEG-4 AVC & 906 & 0.7041 & 777.10 & 0.0318 & 0.1896 \\
\cline { 2 - 7 } & MPEG-4 V & 913 & 0.3819 & 418.04 & 0.0322 & 0.1019 \\
\cline { 2 - 7 } & MPEG-2 & 608 & 0.6953 & 1142.91 & 0.0453 & 0.1900 \\
\hline \multirow{3}{*}{$\begin{array}{l}\text { Promedio Porcen- } \\
\text { tual [\%] }\end{array}$} & MPEG-4 AVC & 0.3733 & 0.3953 & 0.3324 & 0.2907 & 0.3938 \\
\cline { 2 - 7 } & MPEG-4 V & 0.3762 & 0.2144 & 0.1788 & 0.2947 & 0.2117 \\
\cline { 2 - 7 } & MPEG-2 & 0.2505 & 0.3903 & 0.4888 & 0.4152 & 0.3946 \\
\hline
\end{tabular}

Tabla 3. Métricas Escenario 2

\begin{tabular}{|l|l|l|l|l|l|l|}
\hline & \multicolumn{1}{|c|}{ Codec } & $\begin{array}{c}\text { Cantidad de } \\
\text { paquetes }\end{array}$ & $\begin{array}{c}\text { Cantidad } \\
\text { de bytes } \\
\text { [Mbytes] }\end{array}$ & $\begin{array}{c}\text { Tamaño } \\
\text { promedio } \\
\text { paquetes } \\
\text { [bytes] }\end{array}$ & $\begin{array}{c}\text { Tiempo } \\
\text { promedio } \\
\text { intertrama } \\
{[\mathbf{s}]}\end{array}$ & $\begin{array}{c}\text { Velocidad } \\
\text { promedio } \\
\text { [Mbps] }\end{array}$ \\
\hline \multirow{4}{*}{ Promedio general } & MPEG-4 AVC & 906 & 0.7041 & 777.10 & 0.0316 & 0.1896 \\
\cline { 2 - 7 } & MPEG-4 V & 913 & 0.3819 & 418.11 & 0.0322 & 0.1019 \\
\cline { 2 - 7 } & MPEG-2 & 608 & 0.6953 & 1142.91 & 0.0456 & 0.1900 \\
\hline \multirow{3}{*}{$\begin{array}{l}\text { Promedio Porcen- } \\
\text { tual [\%] }\end{array}$} & MPEG-4 AVC & 0.3733 & 0.3953 & 0.3324 & 0.2894 & 0.3938 \\
\cline { 2 - 7 } & MPEG-4 V & 0.3762 & 0.2144 & 0.1788 & 0.2945 & 0.2117 \\
\cline { 2 - 7 } & MPEG-2 & 0.2505 & 0.3903 & 0.4888 & 0.4175 & 0.3945 \\
\hline
\end{tabular}

Tabla 4. Métricas escenario 3

\begin{tabular}{|l|l|l|l|l|l|l|}
\hline & Codec & $\begin{array}{c}\text { Cantidad de } \\
\text { paquetes }\end{array}$ & $\begin{array}{c}\text { Cantidad } \\
\text { de bytes } \\
\text { [Mbytes] }\end{array}$ & $\begin{array}{c}\text { Tamaño } \\
\text { promedio } \\
\text { paquetes } \\
\text { [bytes] }\end{array}$ & $\begin{array}{c}\text { Tiempo } \\
\text { promedio } \\
\text { intertrama } \\
\text { [s] }\end{array}$ & $\begin{array}{c}\text { Velocidad } \\
\text { promedio } \\
\text { [Mbps] }\end{array}$ \\
\hline \multirow{4}{*}{ Promedio general } & MPEG-4 AVC & 906 & 0.7041 & 777.10 & 0.0316 & 0.1896 \\
\cline { 2 - 7 } & MPEG-4 V & 913 & 0.3819 & 418.10 & 0.0321 & 0.1019 \\
\cline { 2 - 7 } & MPEG-2 & 608 & 0.6953 & 1142.91 & 0.0455 & 0.1900 \\
\hline \multirow{3}{*}{$\begin{array}{l}\text { Promedio Porcen- } \\
\text { tual [\%] }\end{array}$} & MPEG-4 AVC & 0.3733 & 0.3953 & 0.3324 & 0.2895 & 0.3938 \\
\cline { 2 - 7 } & MPEG-4 V & 0.3762 & 0.2144 & 0.1788 & 0.2938 & 0.2117 \\
\cline { 2 - 7 } & MPEG-2 & 0.2505 & 0.3903 & 0.4888 & 0.4168 & 0.3945 \\
\hline
\end{tabular}


Tabla 5. Métricas escenario 4

\begin{tabular}{|l|l|l|l|l|l|l|}
\hline \multirow{5}{*}{ Codec } & \multicolumn{1}{|c|}{$\begin{array}{c}\text { Cantidad de } \\
\text { paquetes }\end{array}$} & $\begin{array}{c}\text { Cantidad } \\
\text { de bytes } \\
\text { [Mbytes] }\end{array}$ & $\begin{array}{c}\text { Tamaño } \\
\text { promedio } \\
\text { paquetes } \\
\text { [bytes] }\end{array}$ & $\begin{array}{c}\text { Tiempo } \\
\text { promedio } \\
\text { intertrama } \\
\text { [s] }\end{array}$ & $\begin{array}{c}\text { Velocidad } \\
\text { promedio } \\
\text { [Mbps] }\end{array}$ \\
\hline \multirow{4}{*}{ Promedio general } & MPEG-4 AVC & 906 & 0.7041 & 777.10 & 0.0316 & 0.1896 \\
\cline { 2 - 7 } & MPEG-4 V & 913 & 0.3819 & 418.11 & 0.0320 & 0.1019 \\
\cline { 2 - 7 } & MPEG-2 & 608 & 0.6953 & 1142.97 & 0.0456 & 0.1900 \\
\hline \multirow{3}{*}{$\begin{array}{l}\text { Promedio Porcen- } \\
\text { tual [\%] }\end{array}$} & MPEG-4 AVC & 0.3733 & 0.3953 & 0.3324 & 0.2894 & 0.3938 \\
\cline { 2 - 7 } & MPEG-4 V & 0.3762 & 0.2144 & 0.1788 & 0.2931 & 0.2117 \\
\hline \multirow{2}{*}{$\begin{array}{l}\text { Porcentual relati- } \\
\text { vo al mayor [\%] }\end{array}$} & MPEG-2 & 0.2505 & 0.3903 & 0.4888 & 0.4174 & 0.3945 \\
\cline { 2 - 7 } & MPEG-4 AVC & $99.23 \%$ & $100 \%$ & $67.99 \%$ & $69.30 \%$ & $99.79 \%$ \\
\cline { 2 - 7 } & MPEG-4 & $100 \%$ & $54.24 \%$ & $36.58 \%$ & $70.18 \%$ & $53.63 \%$ \\
\hline
\end{tabular}

Con base en la similitud de valores en los diferentes escenarios, se realizó un análisis de cada métrica para el Escenario 4, que es el más representativo, al tener mayor cantidad de clientes conectados y recibiendo el mismo flujo en forma simultánea. A la tabla 5, representativa del escenario 4, se le han adicionado nuevas filas, para considerar el promedio porcentual respecto al valor mayor obtenido. Estos cálculos permiten una mejor comparación entre los distintos codecs.

El número total de paquetes transmitidos por el servidor y recibidos por cada cliente es prácticamente el mismo en un único codec, para diferentes clientes. No obstante, se puede observar una diferencia en el número de paquetes, para diferentes codecs, cuando se considera la media de todas las PCs para el mismo codec. MPEG-4 Visual utiliza el mayor número de paquetes (913), con una leve diferencia con MPEG-4 AVC (906 paquetes). Por otro lado, para MPEG-2, el número de paquetes fue significativamente menor (un $33.41 \%$ menos), como se observa en la figura 3.

Figura 3. Porcentual relativ-Cantidad de paquetes

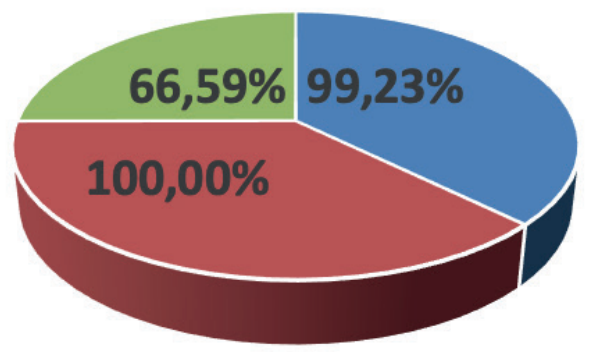

\section{- MPEG-4 AVC \\ - MPEG-4 V \\ - MPEG-2}

El comportamiento del número total de bytes transmitidos por el servidor y recibidos por cada cliente, es prácticamente el mismo en cada codec. El codec MPEG-4 AVC es el que más bytes transmitió (como resultado de un archivo de mayor tamaño), y muestra poca diferencia con los valores de MPEG-2; mientras que el número de bytes transmitidos para MPEG-4 Visual fue, significativamente menor (un $45.76 \%$ menos), como se observa en figura 4. 
Figura 4. Porcentual relativo-Cantidad de bytes

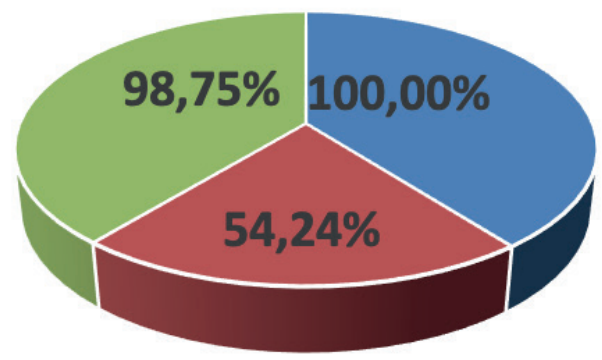

- MPEG-4 AVC

- MPEG-4 V

- MPEG-2

Con respecto al tamaño promedio de los paquetes transmitidos y recibidos por el servidor en cada PC, es prácticamente el mismo en cada codec. Por el contrario, cuando se trata del tamaño promedio de los paquetes, a través de diferentes codecs, MPEG-4 Visual muestra el valor más pequeño (63.42 \% menos en comparación con MPEG-2), representado en la figura 5.

Figura 5. Porcentual relativo-Tamaño de paquetes

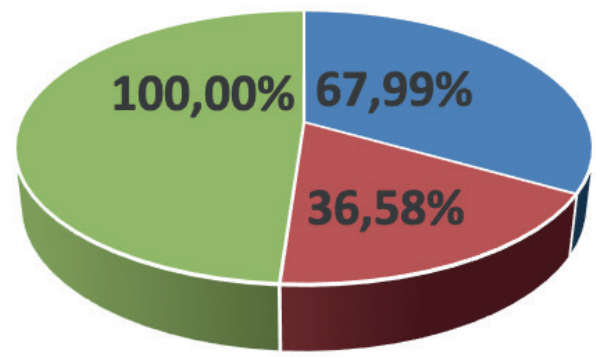

- MPEG-4 AVC

- MPEG-4 V

- MPEG-2

Otro parámetro importante es el promedio de tiempo entre tramas de paquetes transmitidos por el servidor y recibidos en cada PC. Estos son bastante similares para cada codec, para diferentes PCs. Sin embargo, la diferencia se hace evidente, en promedio, entre los tiempos entre tramas de diferentes codecs. Para MPEG-2 el tiempo es mayor, mientras que MPEG-4 AVC muestra el menor tiempo entre tramas (30.7 \% inferior) (figura 6).

Finalmente, al analizar el promedio de la velocidad de transmisión, se observa que es prácticamente el mismo en cada uno de los codecs. Sin embargo, cuando se comparan las velocidades medias entre diferentes codecs, MPEG-4 Visual muestra la tasa de bits más baja (46.37\% inferior) (figura 7).

Figura 6. Porcentual relativo-Tiempo intertrama

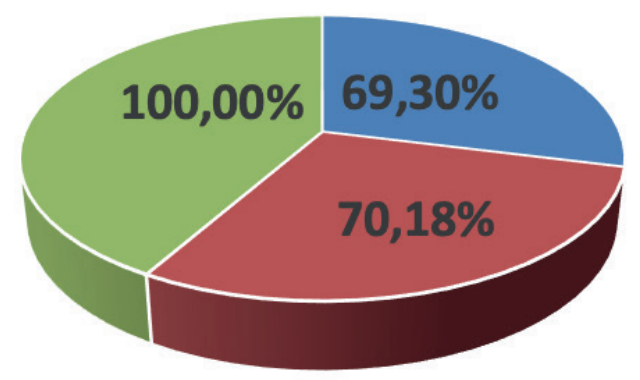

- MPEG-4 AVC

- MPEG-4 V

MPEG-2 
Figura 7. Porcentual relativo-Velocidad

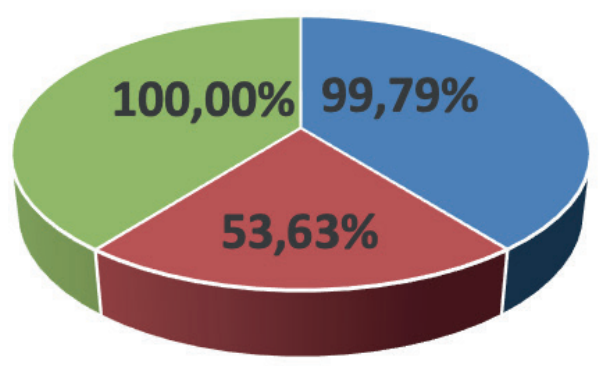

- MPEG-4 AVC

- MPEG-4 V

- MPEG-2

\section{Conclusiones y recomendaciones}

Este estudio experimental contribuye al cuerpo de conocimientos, y proporciona una actualización profesional y vanguardista de los avances en el campo de la comunicación multidifusión, desempeño y calidad de servicio (QoS), en el contexto de la transmisión de video en una verdadera red de laboratorio, como es el caso de una red WAN en un laboratorio de ensayo.

En primer lugar, se examina un conjunto de técnicas y tecnologías, así como el estado del arte para el tráfico de video multidifusión, seguido de una descripción de los escenarios considerados, su topología de red y recursos de hardware. A continuación, se presentan los resultados experimentales en detalle, en forma de diversas mediciones directas, el promedio de las cifras y las estadísticas, de cuatro escenarios con streaming de video con cuatro diferentes codificaciones.

El estudio en el laboratorio de ensayo reveló que:

- Las métricas obtenidas desde el presente laboratorio muestran que, el tráfico de multidifusión de WAN, proporciona la calidad de servicio (QoS) y respuesta esperada en cada estación, en el segmento de red del servidor, así como sobre los segmentos y enlaces compartidos, usando diferentes tipos de codecs, que ofrece una buena visión sobre el comportamiento que cabe esperar de una red real en condiciones similares.

- Las variaciones en las características de las métricas de rendimiento y calidad de servicio (QoS) para el tráfico de multidifusión dependen básicamente del codec de video utilizado.

- El impacto combinado de tráfico global (que incluya otros tipos de tráfico de red), en un enlace de red, dependerá de la topología y los codecs utilizados, así como sobre los ajustes aplicados a dicha topología, mediante los protocolos de multidifusión (OSFP y PIM).

- La adecuada gestión administrativa de la red puede llevar a un uso eficiente de los recursos disponibles, sin comprometer el rendimiento y la QoS esperada.

Los estudios futuros estarán orientados al seguimiento y a la comprensión más profunda de los aspectos presentados en este documento. Una línea de investigación actual añade clientes tipo Wi-Fi IEEE 802.11ac a la topología. En este caso, está previsto explorar otros aspectos de interés, como la introducción de protocolos avanzados, como IPv6 y sus nuevas características para el tráfico de multidifusión. 


\section{Bibliografía}

Abdalla, A., Mazhar, A., Salah, M. y Khalaf, S. (2014). "Comparative Study of Compression Techniques for Synthetic Videos". The International Journal of Multimedia \& Its Applications (IJMA), 6 (2): 1-10. https://dx.doi.org/10.5121/ijma.2014.6201

Addu, R., y Potuvardanam, V. (2014). "Effect of Codec Performance on Video QoE for videos encoded with Xvid, H.264 and WebM/ VP8". Master Thesis, Blekinge Institute of Technology, Karlskrona, Sweden.

Auwera, G., David, P. y Reisslein, M. (2008). "Traffic and quality characterization of single-layer video streams encoded with H.264/ MPEG-4 advanced video coding standard and scalable video coding extension". IEEE Transactions on Broadcasting, 54 (3): 698-718.

Brown, I., Crowcroft, J., Handley, M. y Cain, B. (2002). "Internet Multicast Tomorrow". The Internet Protocol Journal 5 (4): 2-19. https:// ipj.dreamhosters.com/wp-content/uploads/issues/2002/ipj05-4.pdf

Cachinero Pozuelo, J. (2009). "Análisis y modelado de multicast interdominio para el servicio de soportes de video", Proyecto fin de carrera Universidad Politécnica de Madrid. http://oa.upm.es/1840 (accedido el 26 de diciembre de 2019)

Chandrasekar, V. y Baskaran, K. (2012). "Performance of Video Conferencing using Protocol Independent Multicast Routing with Core failure", International Journal of Computer Applications, 41 (10): 22-31. https://doi.org/10.5120/5578-7685

Cisco public white paper (2019). "Cisco Visual Networking Index: Forecast and Trends, 2017-2022". Recuperado de https://www. cisco.com/c/en/us/solutions/collateral/ service-provider/visual-networking-index-vni/white-paper-c11-741490.html (accedido el 26 de diciembre de 2019).

Dai, M., Zhang, Y. y Loguinov, D. (2009). "A unified traffic model for MPEG-4 and H.264 video traces". IEEE Transactions on Multimedia, 11 (5): 1010-1023.

Divxland, (2012). "Video codecs explained". Recuperado de http://www.divxland.org/en/article/5/video_codecs_explained\#.XgUVhUdKiUm (accedido el 26 de diciembre de 2019).

Egger, S. y Braun, T. (2003). "Performance Evaluation of Multicast for Small Conferences", Group Communications and Charges; Technology and Business Models, 5th COST264 International Workshop on Networked Group Communications, NGC 2003, and 3rd International Workshop on Internet Charging and QoS Technologies, ICQT 2003.

Grois, D., Marpe, D., Nguyen, T. y Hadarb, O. (2014). "Comparative Assessment of H.265/MPEG-HEVC, VP9, and H.264/MPEGAVC Encoders for Low-Delay Video Applications". Applications of Digital Image Processing XXXVII, 9217. https://doi. org/10.1117/12.2073323

Hosseini, M., Tanvir Ahmed, D., Shirmohammadi, S. y Georganas, N. (2007). "A Survey of Application Layer Multicast Protocols". IEEE Communications Surveys \& Tutorials 9 (3): 58-74. http://citeseerx.ist.psu.edu/viewdoc/download?doi=10.1.1.70.9653 \&rep=rep1\&type=pdf.

Kenji, F., Masataka, O. y Katsuo, I. (2000). "Integration of Multicast Routing and QoS Routing", Recuperado de https://www.isoc.org/ inet2000/cdproceedings/1c/1c_1.htm (accedido el 26 de diciembre de 2019)

Klare, B. y Burge, M. (2010). "Assessment of H.264 video compression on automated face recognition performance in surveillance and mobile video scenarios", SPIE Defense, Security and Sensing, 7667. https://doi.org/10.1117/12.851349

Lazaris, A. y Koutsakis, P. (2010). "Modeling multiplexed traffic from H.264/AVC videoconference streams". Computer Communications, 33 (10): 1235-1242.

Ludeña, P., Torres, R. Quiñonez, M. y Cueva, S. (2017). "Impacto de la sobrecarga en redes móviles ad-hoc". Revista Enfoque UTE, 8 (4): 16-26. https://doi.org/10.29019/enfoqueute.v8n4

Nakayama, H., Watanabe, Y. y Higashi, A. (2008). "H264/AVC HDTV Video codec LSI". Fujitsu Sci. Tech. J., 44 (3): $351-358$.

Pérez, S., Facchini, H., Dantiacq, A., Cangemi, G. y Campos, J. (2015). "An Evaluation of QoS for intensive video traffic over 802.11e WLANs-Quantitative analysis". 25th Conference on Electronics, Communications and Computers CONIELECOMP 2015, 8-15. https://doi.org/10.1109/CONIELECOMP.2015.7086933

Sun, Y., Sheriff, I., Belding-Royer, E. y Almeroth, K., (2005). "An Experimental Study of Multimedia Traffic Performance in Mesh Networks". International Workshop on Wireless Traffic Measurements and Modeling, 25-30.

Tanwir, S. y Perros, H. (2013). "A survey of VBR video traffic models". IEEE Communications Surveys and Tutorials, 15 (4): 1778-1802.

Tanwir, S., Perros, H. y Anjum, B. (2014). "A QoS evaluation of video traffic models for H.264 AVC video". Fifth International Conference on Next Generation Networks and Services, 313-320.

Torres, J., Ramírez, R. y López, D., (2011). "Estado del arte de IPTV y consideraciones técnicas para su migración a IPv6 en Colombia". Revista Redes de Ingeniería, 2 (1): 45-64. https://doi.org/10.14483/2248762X.7179

Ueno, S., Kato,. y Suzuki, K., (2000). "Analysis of Internet Multicast Traffic Performance Considering Multicast Routing Protocol". 2000 International Conference on Network Protocols, 95-104. https://ieeexplore.ieee.org/stamp/stamp.jsp? $\mathrm{tp}=\&$ arnumber $=896286$

Unreal Streaming Technologies. "Unreal Media Server". Recuperado de http://umediaserver.net/ (accedido el 26 de diciembre de 2019)

Seeling, P., Reisslein, M. y Kulapala, B., (2004). "Network performance evaluation with frame size and quality traces of single-layer and two-layer video: A tutorial". IEEE Communications Surveys and Tutorials, 6 (3): 58-78.

Shin, Y., Choi, M., Koo, J., Kim, Y., Ihm, J. y Choi, S., (2011). "Empirical Analysis of Video Multicast over WiFi". Third International Conference on Ubiquitous and Future Networks (ICUFN), 381-386.

Villarreal, M., Mariño, J., Márquez, J. y Nuñez, L. (2019). "Evaluación de una red inalámbrica de banda ancha para VolP". Revista Enfoque UTE, 10 (4): 28-44. https://doi.org/10.29019/enfoque.v10n4

Video base utilizado. Recuperado de https://www.youtube.com/watch?v=iclkHol4L98 (accedido el 26 de diciembre de 2019).

Wireshark network protocol analyzer. Recuperado de https://www.wireshark.org (accedido el 26 de diciembre de 2019). 